\title{
Les télévisions celtiques TG4, S4C, BBC Alba, France 3 Bretagne, Brezhoweb : état des lieux et enjeux
}

\section{Pierre Costecalde}

\section{(2) OpenEdition}

Journals

\section{Édition électronique}

URL : https://journals.openedition.org/etudesirlandaises/8222

DOI : 10.4000/etudesirlandaises.8222

ISSN : 2259-8863

\section{Éditeur}

Presses universitaires de Caen

\section{Édition imprimée}

Date de publication : 31 décembre 2019

Pagination : 59-75

ISBN : 978-2-84133-964-8

ISSN : 0183-973X

\section{Référence électronique}

Pierre Costecalde, «Les télévisions celtiques TG4, S4C, BBC Alba, France 3 Bretagne, Brezhoweb : état des lieux et enjeux », Études irlandaises [En ligne], 44-2 | 2019, mis en ligne le 06 mai 2020, consulté le 15 novembre 2022. URL : http://journals.openedition.org/etudesirlandaises/8222 ; DOI : https:// doi.org/10.4000/etudesirlandaises.8222

\section{(c) (†) (-)}

Creative Commons - Attribution - Pas d'Utilisation Commerciale - Partage dans les Mêmes Conditions 4.0 International - CC BY-NC-SA 4.0

https://creativecommons.org/licenses/by-nc-sa/4.0/ 


\section{Les télévisions celtiques TG4, S4C, BBC Alba, France 3 Bretagne, Brezhoweb : état des lieux et enjeux}

Résumé: Les chaînes de télévision en langue celtique TG4 (Irlande), S4C (Pays de Galles), BBC Alba (Écosse), France 3 Bretagne (pour ses émissions en breton) et Brezhoweb (France, Bretagne) reposent sur des langues dont la démographie linguistique reste préoccupante. Dans un contexte de très forte concurrence et dans un mediascape hyper-fragmenté, elles constituent des pôles de résistance implantés dans l'espace des lieux émettant d'abord pour leurs propres communautés linguistiques. Dans la mesure où elles dépendent des flux financiers (financescape) et de subventions, elles sont l'objet d'une tension permanente entre économie et médias. La récente crise financière et économique a provoqué la diminution ou la stagnation de leurs budgets qui dépendent d'un audimat fluctuant. La majorité des chaînes déterritorialisent désormais certaines de leurs émissions grâce à Internet. Elles augmentent ainsi leur audience à la fois dans leur communauté respective et dans le grand public, grâce au sous-titrage optionnel et à l'adaptation de formats internationaux comme le soap opera ou la sitcom. Vecteurs de convergence, les chaînes sont disponibles sur de nombreux supports numériques. Leur avenir va, par conséquent, dépendre des décisions de l'après-Brexit au Royaume-Uni, de la mise en œuvre du grand marché numérique européen et, surtout, de l'attitude des jeunes générations envers les langues, de leur apprentissage et de l'utilisation qu'elles vont en faire sur les multiples plateformes numériques.

Mots clés : télévision, langues celtiques, audimat, communautés linguistiques, territorialisation, déterritorialisation, sous-titrage, convergence, espace des lieux et des flux, séries.

Abstract: The television channels broadcasting in a Celtic language TG4 (Ireland), S4C (Wales), BBC Alba (Scotland), France 3 Bretagne's programmes in Breton and Brezhoweb (France/Bretagne) rest on languages whose linguistic demographics is worrying. Faced with a very strong competition in a hyper-fragmented mediascape, they are centres of resistance against globalisation, located in the "space of places" broadcasting for their language communities. Their dependence on financial flows creates a permanent tension between economy and media. The financial and economic crisis has triggered a reduction or a stagnation of these channels' budgets. Most of them are now deterritorialising some of their programmes through the Internet. Therefore, they increase their audience rates among their diasporas and the public at large by resorting to optional subtitles and adapting to local audiences international formats such as soap operas or sitcoms. Part and parcel of convergence, these channels are available on numerous digital platforms. Their future will depend on a postBrexit agreement, on the implementation of the European Digital Single Market, but above all, on the younger generation's approach to learning Celtic languages and their use on the numerous audio-visual digital platforms.

Keywords: television, Celtic languages, audience ratings, linguistic communities, territorialisation, deterritorialisation, subtitling, convergence, space of places and space of flux, series. 
L'espace médiatique européen ou mediascape ${ }^{1}$ est de plus en plus traversé par les flux des chaînes de télévision. Selon Mavise ${ }^{2}$, pas moins de 11336 chaînes (publiques ou commerciales) sont en concurrence. 238 chaînes peuvent être captées en Irlande, 567 au Royaume-Uni et 817 en France. Dans un tel environnement médiatique, TG4 en Irlande, S4C au Pays de Galles, BBC Alba en Écosse, France 3 Bretagne et ses émissions en breton ainsi que Brezhoweb (chaîne en langue bretonne sur Internet) font face à une très forte concurrence dans leur pays respectif. On peut donc, à juste titre, se demander quelle est l'influence de ces chaînes sur les audimats des communautés linguistiques qu'elles défendent. Selon Mike Cormack ${ }^{3}$, cet impact n'est pas du tout évident. Joshua Fishman souligne, pour sa part, que les mass media sont moins importants que la transmission intergénérationnelle, dans la famille, une communauté linguistique ou l'éducation ${ }^{4}$. Cependant selon Eithne O’Connell, les médias sont de plus en plus présents dans tous les domaines qu'ils relient entre eux de façon plus complexe ${ }^{5}$. Afin d'évaluer l'influence de ces télévisions il faut d'abord estimer leur diffusion dans un territoire donné, pour une communauté linguistique déterminée: il s'agit d'une territorialisation des flux télévisés de chaînes qui émettent dans un espace des lieux, concept mis en avant par Manuel Castells.

\section{Territorialisation des chaînes en langue celtique et espace des lieux}

Selon ce dernier, l'espace des lieux privilégie l'interaction sociale et l'organisation des institutions (dont les médias) sur la base d'une proximité physique: «Most human experience, and meaning, are still locally based ${ }^{6}$. Les chaînes de télévision celtiques qui émettent dans l'espace des lieux vont donc être appelées à se territorialiser et à émettre dans leur pays respectif afin de pouvoir atteindre les locuteurs en langue minoritaire qui sont l'objet de la mission première de ces chaînes. Il va donc y avoir territorialisation des flux télévisés au sens où l'entend Jean Laponce: "The first, the dominant, the cardinal rule that should guide a minority language is the territorial

1. Arjun Appadurai, Après le colonialisme: les conséquences culturelles de la globalisation, Françoise Bouillot (trad.), Paris, Payot \& Rivages, 2005, p. 73.

2. Mavise («Database on TV channels, on-demand services and licenses in Europe»), http://mavise. obs.coe.int/2013.

3. Mike Cormack, «The Media and Language Maintenance», in Minority Language Media: Concepts, Critiques and Case Studies, Mike Cormack, Niamh Hourigan (dir.), Clevedon, Multilingual Matters, 2007, p. 58.

4. Joshua A. Fishman, «From Theory to Practice (and Vice Versa): Review, Reconsideration and Reiteration", in Can Threatened Languages Be Saved? Reversing Language Shift, Joshua A. Fishman (dir.), Clevedon, Multilingual Matters, 2000, p. 451-452.

5. Eithne O’Connell, «Towards a Template for a Linguistic Policy for Minority Language Broadcasters", in Social Media and Minority Languages. Convergence and the Creative Industries, Elin Haf Gruffydd Jones, Enrique Uribe-Jongbloed (dir.), Bristol, Multilingual Matters, 2013, p. 188.

6. Manuel Castells, The Information Age: Economy, Society, and Culture, vol. II, The Power of Identity, $2^{\mathrm{e}}$ éd., Chichester, Wiley-Blackwell, 2010, p. 181. 
principle: territorialize and keep territorializing» ${ }^{7}$. Le premier obstacle rencontré par une chaîne celtique est donc d'ordre démographique et conditionne la survie culturelle et économique de la chaîne.

La démographie linguistique est donc une donnée essentielle pour les chaînes car chacune émet d'abord pour une communauté linguistique déterminée. Nous allons donc examiner chacune de ces démographies et l'audimat des chaînes par rapport à chacune d'elles. En République d'Irlande, sur une population de 4761865 habitants, 1761420 ont répondu lors du dernier recensement de 2016 qu'ils étaient capables de parler l'irlandais ${ }^{8}$. En 2011, ils étaient 1774437 , soit une légère diminution de $0,7 \%{ }^{9}$. En 2011, lors du dernier recensement mené au Royaume-Uni, sur 1735711 habitants que comptait l'Irlande du Nord, 184898 soit $10,65 \%$ ont déclaré avoir une certaine connaissance de l'irlandais tandis que $3,74 \%$ ont prétendu le comprendre, le parler, le lire et l'écrire ${ }^{10}$. Lors du recensement de 2001, 10,4\% de la population âgée de 3 ans et plus disaient posséder une certaine connaissance de la langue tandis que $4,6 \%$ prétendaient être capables de lire, d'écrire, de parler et de comprendre l'irlandais ${ }^{11}$. On retrouve à peu près les mêmes tendances au Pays de Galles et en Écosse.

Au Pays de Galles, lors du dernier recensement de 2011, sur une population totale de 3100000 habitants, $19 \%$ soit 562000 habitants âgés de 3 ans et plus ont déclaré qu'ils pouvaient parler le gallois. En 2001, ils étaient 576000 soit une baisse de $2 \%$ depuis le recensement de $2011^{12}$. En Écosse, le recensement de 2011 sur

7. Jean Laponce, «The Governance of Minority Languages: Principles and Exceptions », communication à la conférence «Debating Language Policies in Canada and Europe», université d'Ottawa, 31 mars-2 avril 2005, p. 2, en ligne: http://socialsciences.uottawa.ca/crfpp/sites/socialsciences. uottawa.ca.crfpp/files/laponce.pdf.

8. Central Statistics Office, Census 2016: Summary Results - Part 1, Dublin, Central Statistics Office, avril 2017, p. 8 et 66, en ligne: https://www.cso.ie/en/csolatestnews/presspages/2017/ census2016summaryresults-part1.

9. Central Statistics Office, This is Ireland. Highlights from Census 2011, Part 1, Dublin, Central Statistics Office, mars 2012, p. 40, en ligne: https://www.cso.ie/en/media/csoie/census/documents/ census2011pdr/Census_2011_Highlights_Part_1_web_72dpi.pdf; Central Statistics Office, Census 2016..., p. 66.

10. Northern Ireland Statistics and Research Agency, Northern Ireland Neighbourhood Information Service, «Census 2011 », «Irish Language Skills», en ligne: https://www.ninis2.nisra.gov.uk/public/ Theme.aspx?themeNumber $=136 \&$ themeName $=$ Census +2011 ; Northern Ireland Statistics and Research Agency, Northern Ireland Census 2011. Key Statistics Summary Report, National Statistics publication, septembre 2014, p. 75, Table KS209NI: «Table population: All usual residents aged 3 and over», en ligne: https://www.nisra.gov.uk/sites/nisra.gov.uk/files/publications/2011-censusresults-key-statistics-summary-report.pdf; «Proportion of the population of Northern Ireland with some langage skills in Irish or Ulster-Scots in 2011 », portail en ligne Statista, https://www.statista. com/statistics/329781/census-population-northern-ireland-irish-ulster-scots-language ability.

11. Northern Ireland Statistics and Research Agency, 2001 Census. Key Statistics for Settlements Tables, p. 201, Table KS24, en ligne: https://www.nisra.gov.uk/sites/nisra.gov.uk/files/publications/2001census-results-key-statistics-for-settlements-report-tables.pdf.

12. Office for National Statistics, «2011 Census », «Key Statistics for Wales, March 2011 », 11 décembre 2012, en ligne: https://www.ons.gov.uk/peoplepopulationandcommunity/populationandmigration/ populationestimates/bulletins/2011censuskeystatisticsforwales/2012-12-11. 
5295403 habitants a montré que 58000 personnes (ou 1,1 \% de la population) âgées de 3 ans et plus se disent capables d'utiliser le gaélique écossais. Ce total accuse une légère chute de 1,2 \% (59000 gaélophones) par rapport au recensement de $2001^{13}$.

En Bretagne, au $1^{\mathrm{er}}$ janvier 2016, la population comptait 3306529 habitants $^{14}$. En 2007, l'Office public de la langue bretonne, se fondant sur un sondage de l'institut TMO-Régions commandé par Fañch Broudic, estimait le nombre des bretonnants actifs de plus de 2 ans à $206000^{15}$. En 2018, l'Office public de la langue bretonne, se fondant sur un sondage de l'institut TMO-Régions pour le conseil régional de Bretagne et sur ses propres estimations, estimait le nombre de bretonnants à 225000 et le nombre de locuteurs passifs à $125000^{16}$.

Au total, même si les données démographiques ne sont pas toujours fiables ( «untrustworthy», comme le note Cathair Ó Dochartaigh ${ }^{17}$ ), la majeure partie de ces langues accusent une légère baisse démographique en dépit des efforts entrepris dans les domaines éducatif et législatif par la plupart des gouvernements et du soutien apporté aux langues régionales et minoritaires par la Charte européenne des langues régionales et minoritaires ${ }^{18}$. L'indice d'écoute, l'audimat, va mesurer l'impact de la chaîne à la fois sur une communauté de locuteurs en langue celtique et dans tout le reste du pays. Nous aurons donc, quand la chaîne consent à les fournir, deux types de données: celles de la chaîne dans la communauté linguistique dans l'espace des lieux et celles correspondant à tout le pays, dans un espace plus large mais territorialisé.

La chaîne en langue irlandaise de service public, TG4, a commencé d'émettre le 31 octobre 1996 après une longue campagne ${ }^{19}$. En 2017, le taux de pénétration journalier et hebdomadaire de la chaîne dans la communauté irlandophone était de $90 \%$. En 2016, elle était de $92 \%$ soit une baisse de $2 \%{ }^{20}$. Le taux de pénétration (reach) qui peut être journalier, hebdomadaire, mensuel ou annuel désigne de façon générale la part de la population ou d'un groupe-cible déterminé qui, à un

13. National Records of Scotland, «Census 2011: Key Results on Population, Ethnicity, Identity, Language, Religion, Health, Housing and Accommodation in Scotland - Release 2A», en ligne: https://www. nrscotland.gov.uk/news/2013/census-2011-release-2a?_ga=2.9918197.1998649709.1556444023520621390.1556444023.

14. Institut national de la statistique et des études économiques, «Populations légales 2016. Recensement de la population: régions, départements, arrondissements, cantons et communes», en ligne: https://www.insee.fr/fr/statistiques/3677855.

15. Office public de la langue bretonne, «Situation sociolinguistique», en ligne: http://www.fr.brezhoneg. bzh/6-situation-sociolinguistique.htm.

16. Office public de la langue bretonne, «Les chiffres clés de la langue bretonne», en ligne: http:// www.fr.brezhoneg.bzh/5-chiffres-cles.htm.

17. Cathair Ó Dochartaigh, "The Irish Language, Historical and Social Perspective», in The Celtic Languages, Donald MacAulay (dir.), Cambridge, Cambridge University Press, 1992, p. 25.

18. Conseil de l'Europe, Application de la Charte européenne des langues régionales ou minoritaires, 3 mars 2014, doc. 13436, en ligne: https://www.coe.int/t/dg4/education/minlang/sgreports/ SGReport2013_fr.pdf.

19. Niamh Hourigan, Escaping the Global Village: Media, Language, and Protest, Oxford, Lexington Books, 2004, p. 97.

20. TG4, Annual Report 2017, p. 9, en ligne: https://dlog0s8nlbd0hm.cloudfront.net/tg4-redesign-2015/ wp-content/uploads/2015/08/TG4-Tuarascail-17-B1.pdf. 
moment ou durant une période donnée, a regardé une émission de télévision. Le taux d'audience (ou share) nationale, journalière, moyenne était en 2017 de 1,90 \% ${ }^{21}$ contre $1,80 \%$ en $2016^{22}$ soit une progression de $5 \%{ }^{23}$. Le taux d'audience est le pourcentage de tous les spectateurs d'une chaîne sur une période donnée ${ }^{24}$. Les données fournies par TG4 sur l'audimat en Irlande du Nord sont très irrégulières. En 2016, le rapport annuel de la chaîne mentionne que le taux de pénétration hebdomadaire de la chaîne était de $7 \%{ }^{25}$. TG4 reste la septième chaîne la plus regardée en Irlande. On retrouve la même dichotomie entre pénétration dans la communauté linguistique et part d'audience globale au Pays de Galles.

S4C (en gallois Sianel Pedwar Cymru) est une chaîne de service public créée le $1^{\text {er }}$ novembre 1982. Sa mission première est d'émettre pour les gallophones. Si l'on considère l'audimat de la chaîne en 2017/2018, on remarque que 166000 gallophones ont regardé S4C chaque semaine. Ils étaient 173000 en 2016/2017. Chaque semaine 365000 téléspectateurs non gallophones ont regardé la chaîne (ils étaient 347000 en 2016/2017). Au total, 9347000 téléspectateurs ont regardé S4C pendant l'année 2017/2018 dans l'ensemble du Royaume-Uni ${ }^{26}$.

BBC Alba a commencé d'émettre le 19 septembre 2008. Elle est administrée en partenariat avec la BBC. Le taux de pénétration annuel de la chaîne en 2016/2017 parmi les spectateurs gaélophones était de $67 \%$. En 2017/2018, il s'élevait à 62,1\%. Son taux de pénétration national annuel était de $11,3 \%$ en $2017 / 2018$ et de $13 \%$ en $2016 / 2017^{27}$. Toutefois, l'image de BBC Alba reste élevée pour $82 \%$ des gens interrogés et le taux de satisfaction pour les émissions a été de $8,2 \%$ pour les gaélophones et de 7,2 \% pour les autres téléspectateurs sur le plan national ${ }^{28}$. Ces trois chaînes celtiques accusent donc une légère réduction de leur audience dans les communautés de locuteurs. Elle est compensée, nous le verrons, par une augmentation du visionnement des chaînes sur d'autres plateformes numériques. Toutes ces données sont publiées chaque année par les différentes chaînes celtiques en Irlande et au Royaume-Uni. Il en va autrement des deux chaînes françaises qui ne publient pas de rapport public sur leurs audimats et sur leurs budgets respectifs qui restent très confidentiels. Ces derniers ne peuvent être obtenus que difficilement.

21. Ibid., p. 20.

22. TG4, Annual Report 2016, p. 9, en ligne: https://dlog0s8nlbd0hm.cloudfront.net/tg4-redesign-2015/ wp-content/uploads/2015/08/TG4-Tuarascail-16-B1.pdf.

23. TG4, Annual Report 2017, p. 20.

24. Television Audience Measurement Ireland, "Share», in "Glossary», en ligne: https://www. tamireland.ie/education/glossary.

25. TG4, Annual Report 2016, p. 10.

26. S4C, Annual Report and Statement of Accounts for the 12 Month Period to 31 March 2018, p. 46, en ligne: https://dlo6cycw1kmbs.cloudfront.net/media/media_assets/s4c-annual-report-andaccounts-spreads.pdf.

27. MG Alba, Annual Report and Statement of Accounts 2016/2017, p. 26, en ligne: http://www.mgalba. com/downloads/reports/annual-report-16-17.pdf; et MG Alba, Annual Report and Statement of Accounts 2017/2018, p. 40, en ligne: http://www.mgalba.com/downloads/reports/annualreport-17-18.pdf.

28. MG Alba, Annual Report and Statement of Accounts 2016/2017, p. 26. 
Deux responsables des émissions en langue bretonne de France 3 Bretagne nous ont cependant fourni, lors d'entretiens, un certain nombre de données concernant les émissions en langue bretonne. Selon Bernard Leroux, ancien responsable des émissions en langue bretonne:

En gros on fédère entre 15 et 20000 spectateurs... spectateurs, oui, et voilà, vous avez là une courbe, alors le breton est en noir, c'est assez irrégulier, de façon très curieuse, on ne sait pas trop d'où ça vient parce qu'on essaie de comprendre s'il y a un systématisme ou pas ${ }^{29}$.

Maël Le Guennec, nouveau responsable des émissions en langue bretonne depuis janvier 2014, précise:

Donc nous, les émissions en langue bretonne, on a un socle d'audience. En gros, c'est une moyenne de 17000 personnes sur les dernières années, c'est notre socle d'audience, c'est-à-dire qu'on sait que tous les dimanches on aura au moins environ ça ${ }^{30}$.

Certaines données doivent auparavant être expliquées. Ainsi, le taux d'audience moyen (TM), tel qu'il est défini par Médiamétrie, désigne la part de la population française de plus de 15 ans qui regarde une émission de télévision ou écoute une station de radio. Un taux moyen d'audience de $10 \%$ signifie donc qu'une personne sur dix de plus de 15 ans a regardé une chaîne ou une émission pour un moment ou une période donnée. Le taux moyen est une donnée différente de la part d'audience. En télévision, pour mesurer l'audience d'un programme, le critère le plus utilisé reste le taux moyen, aussi appelé audience moyenne.

La part d'audience (PDA) permet de mesurer une audience en tenant compte du contexte concurrentiel. Exemple: 10 millions de Français regardent la télévision à un instant T. Si France 3 en capte 1 million, la chaîne affichera $10 \%$ de parts de marché. Si M6 en engrange 2 millions, elle atteindra $20 \%$. TVSP exprime le nombre de téléspectateurs en milliers ${ }^{31}$.

La part de marché et le taux d'audience des émissions en langue bretonne varient selon le jour, l'heure de la diffusion et le genre des émissions. Ainsi, l'émission d'information de France 3 Bretagne, An Taol Lagad (Le coup d'œil), diffusée du lundi au vendredi, affiche un nombre de téléspectateurs qui varie entre 18000 et $37000^{32}$, a un taux d'audience moyen (TM) entre $0,6 \%$ et 1,2 \%, et une part d'audience moyenne (PDA) entre $5 \%$ et 7,3\%. Bali Breizh (Boulevard de Bretagne) entre 2014 et fin 2017 a une part d'audience moyenne qui évolue entre $4,6 \%$ et $3 \%$ et un TVSP (téléspectateurs en milliers) entre 21 et 11. Mouchig Dall (Colin maillard) affiche une PDA entre $2,43 \%$ et $1 \%$. Le magazine régional d'actualités a une PDA entre $2,9 \%$ et $3,2 \%$ tandis que le journal régional se situe pour le PDA entre $9,35 \%$ et $19 \%$.

29. Entretien avec Bernard Leroux, France 3 Bretagne, 4 février 2013.

30. Entretien avec Maël Le Guennec, France 3 Bretagne, 2 octobre 2014.

31. Médiamétrie, «Les mots des médias», page consultée sur le site de Médiamétrie le 12 avril 2016, désormais inaccessible.

32. Données aimablement fournies par Maël Le Guennec, France 3 Bretagne, le 15 mai 2015. 
Il faut aussi citer la sitcom Breizh Kiss (Baiser de Bretagne). Si on examine la part de marché prise par l'émission du 13 juillet au 24 août 2014, on remarque que son TM pour les mois de juillet et d'août 2014 s'élève à $8 \%$ et sa PDA à $7 \%$. Cette sitcom occupe la quatrième place, en Bretagne, après TF1, France 2 et M6 et la septième place en France pour la même période. Elle a été rediffusée en continu pendant les vacances d'été 2014 et a vu alors sa PDA augmenter.

La dernière télévision dont l'audimat doit être mentionné est Brezhoweb. Le directeur de cette télévision sur Internet, Lionel Buannic, explique qu'elle est de plein exercice et a été créée en 2010. En octobre 2010, la chaîne a signé une convention avec le Conseil supérieur de l'audiovisuel qui a été renouvelée en 2015 et en 2018. Lionel Buannic explique: "On est une véritable chaîne de télévision à part entière avec des programmes qui changent en permanence ${ }^{33}$. Elle n'émet que sur Internet. Sa place est spéciale dans le mediascape car la chaîne diffuse ses émissions à la fois dans l'espace des lieux et au-delà dans l'espace des flux. Ceci explique que son audimat sera examiné dans la dernière partie consacrée à l'espace des flux. Lionel Buannic qualifie Brezhoweb de «chaîne hybride», c'est-à-dire ayant les mêmes obligations qu'une chaîne traditionnelle non connectée mais sur Internet. Brezhoweb est donc à la fois territorialisée et déterritorialisée.

L'audimat conditionne en grande partie le budget ou la subvention versée aux chaînes. Belinda Albrecht résume la situation de dépendance ainsi:

Language media is dependent on funding. "Bad" statistics can have a direct negative effect on receiving funding. But exact and available statistics are the prerequisite to argue in favour of the importance of a given media service. The classical apologising answer "these are very low figures but..." is still heard too often. 1,000 people watching TV in a minority language do not equate zero viewers ${ }^{34}$.

Toutes les chaînes ont souffert après la crise économique de 2008. Elles sont toutes dépendantes des marchés globaux du financescape qu'Arjun Appadurai définit comme un paysage mystérieux et difficile à déchiffrer. C’est, pour lui,

[...] les marchés de change, les bourses nationales et les spéculations sur les biens et les services [qui] font passer, à la vitesse de la lumière, des sommes colossales à travers les tourniquets nationaux; chaque petite différence de point et d'unité de temps pouvant avoir d'immenses implications ${ }^{35}$.

Les flux financiers du financescape qui font partie de l'espace des flux sont considérés comme les flux dominants. Toutes les chaînes de télévision en dépendent. David Morley et Kevin Robins reconnaissent que: "This new space of flows is

33. Entretien avec Lionel Buannic, directeur de Brezhoweb, 21 novembre 2013.

34. Belinda Albrecht, "The Potentials of Launching Digital Minority Language Media: Brezhoweb - the Web TV in Breton", Res celticae, 2014, p. 163-182.

35. Arjun Appadurai, Après le colonialisme..., p. 73. 
shaped and controlled by transnational capital ${ }^{36}$. C'est dans l'espace des flux que les chaînes sont déterritorialisées.

\section{Déterritorialisation des chaînes et espace des flux}

Les flux financiers qui dominent l'espace des flux ont un impact sur toutes les chaînes de télévision et en particulier sur les chaînes de télévision en langue celtique qui, selon Helen Kelly-Holmes:

[...] must learn to compete, not with other languages or for government attention, but instead with all sorts of other niche markets, all of which face equal threat if they do not meet the criteria of the "market"

En effet, d'après Rosemary Day, depuis les années 1980, la seule logique qui guide les médias a été le marché ${ }^{38}$. Karen Arriaza Ibarra, Eva Nowak et Raymond Kuhn confirment cette évolution quand ils écrivent: «PSM [Public Service Media] need to attract audiences for reasons of funding and political legitimacy ${ }^{39}$. Le budget et les subventions des chaînes celtiques dépendent de leur audimat et de leur part de marché. Selon Pierre Bourdieu: «La télévision subit, plus que tous les autres univers de production culturelle, la pression du commerce par l'intermédiaire de l'audimat ${ }^{40}$. Le budget de toutes les chaînes celtiques dépend aussi du financescape, des flux financiers dominants. En effet, la crise financière et économique de 2008 a contraint les gouvernements à réduire les subventions accordées aux chaînes dont le budget a été réduit ou tout simplement gelé. Nous citerons à titre d'exemple les budgets des chaînes pour 2008 et 2017. Ceux-ci comprennent des revenus commerciaux (publicité, ventes, locations) qui, eux aussi, ont subi les conséquences de la crise.

Le budget de TG4 en 2008 était de $35473000 €$. En 2017, il s'élève à $32790000 €$. Les revenus commerciaux sont passés, après de nombreuses variations, de $4030000 €$ en 2008 à $4070000 €$ en $2017^{41}$. L'on observe le même phénomène de baisse avec S4C. La chaîne est désormais financée par la redevance télévisuelle. En 2008 sa subvention s'élevait à $98440000 £$ et ses revenus commerciaux à $4071000 £$. En

36. David Morley, Kevin Robins, Spaces of Identity: Global Media, Electronic Landscapes and Cultural Boundaries, Londres, Routledge, 1995, p. 38.

37. Helen Kelly-Holmes, "Foreword", in Minority Language Broadcasting. Breton and Irish, Helen Kelly-Holmes (dir.), Clevedon, Multilingual Matters, 2001, p. 3.

38. Rosemary Day, in «Debate: The Context of Minority Language Broadcasting», in Minority Language Broadcasting..., p. 49.

39. Karen Arriaza Ibarra, Eva Nowak, Raymond Kuhn, «Introduction: The Relevance of Public Service Media in Europe", in Public Service Media in Europe: A Comparative Approach, Karen Arriaza Ibarra, Eva Nowak, Raymond Kuhn (dir.), Londres, Routledge, 2015, p. 5.

40. Pierre Bourdieu, Sur la télévision, suivi de L'emprise du journalisme, Paris, Raison d'agir, 1996, p. 45.

41. TG4, Annual Report 2008, p. 62 et 55, en ligne: https://d1og0s8nlbd0hm.cloudfront.net/tg4redesign-2015/wp-content/uploads/2015/08/TG4-Tuarascail-08-B.pdf; et TG4, Annual Report 2017, p. 48 et 16 . 
$2016 / 2017$, sa subvention a été réduite à $81262000 £$ et ses revenus commerciaux à $2208000 \mathfrak{E}^{42}$.

BBC Alba se trouve aussi dans une situation identique. La subvention accordée d'abord par le gouvernement britannique et ensuite par le gouvernement écossais n'a que très peu évolué. En 2008/2009, elle s'élevait à la somme de $12100000 \mathrm{E}$ sans aucun autre revenu supplémentaire. En 2017/2018, elle était de $12800000 £$, à laquelle s'ajoutent d'autres revenus (autres subventions et locations de studios), soit $348,5 \mathrm{k} \mathfrak{E}$ mais $280,3 \mathrm{k} \mathfrak{E}$ en $2016 / 2017^{43}$. La faible hausse est due à la très forte concurrence entre chaînes. Une lecture de tous les rapports de TG4, S4C et BBC Alba révèle une très grande insatisfaction face à la stagnation des subventions car, selon leurs responsables, elles nuisent au développement des chaînes et à la qualité des émissions. En Irlande, le Broadcasting Authority of Ireland (BAI) dans son rapport de 2018 recommande que la subvention de TG4 soit augmentée: «The BAI believes that both funding increases are justified, as, in its view, the funding currently available is not sufficient to ensure the broadcaster's sustainability» ${ }^{44}$. L'enquête indépendante demandée par le gouvernement britannique aboutit aux mêmes conclusions:

This lack of certainty on the grant-in-aid has created a disproportionate level of anxiety and uncertainty among S4C and stakeholders that goes well beyond the impact of the actual sums involved ${ }^{45}$.

BBC Alba exprime les mêmes réserves sur son budget. Selon son directeur général:

The seriously constrained nature of $B B C A L B A$ 's annual content budget - around $£ 11 \mathrm{~m}$ from MG ALBA and between $£ 6 \mathrm{~m}$ and $£ 7 \mathrm{~m}$ from the $B B C$ - continued to act as a serious barrier to addressing the challenges facing the channel ${ }^{46}$.

France 3 Bretagne et Brezhoweb font aussi face aux mêmes difficultés budgétaires. Le budget des émissions en langue bretonne de France 3 Bretagne (redevance + subvention) s'élevait en 2012 à $2555 \mathrm{k} €$, en 2013 il était de $2645 \mathrm{k} €$ et en 2014 de $2500 \mathrm{k} €$. La subvention de l'état soit $185 \mathrm{k} €$ n'a pas bougé sur les trois ans ${ }^{47}$.

42. S4C, Annual Report 2008, p. 111, en ligne: http://www.s4c.cymru/abouts4c/annualreport/acrobats/ adroddiad-blynyddol-s4c-annual-report-2008.pdf; et S4C, Annual Report and Statement of Accounts for the 12 Month Period to 31 March 2017, p. 115, en ligne: http://www.s4c.cymru/abouts4c/ annualreport/acrobats/s4c-annual-report-2017.pdf.

43. MG Alba, Annual Report and Statement of Accounts 2008/2009, p. 34, en ligne: http://www.mgalba. com/downloads/reports/annual-report-08-09.pdf; MG Alba, Annual Report and Statement of Accounts 2017/2018, p. 22; et MG Alba, Annual Report and Statement of Accounts 2016/2017.

44. Broadcasting Authority of Ireland, Scannain. Irish for Movies, en ligne: https://scannain.com/ irish/industry/bai-funding-review-2018.

45. Euryn Ogwen Williams, Department for Digital, Culture, Media and Sport, Building an S4C for the Future, An Independent Review, décembre 2017, p. 8, en ligne: https://www.gov.uk/government/ publications/building-an-s4c-for-the-future-an-independent-review-by-euryn-ogwen-williams.

46. "Chief Executive's Review», in MG Alba, Annual Report and Statement of Accounts 2017/2018, p. 13.

47. Données communiquées par Maël Le Guennec, France 3 Bretagne, le 12 mai 2015. 
Cependant la situation financière d'une chaîne n'explique pas tout. Comme le concède Alan Esslemont, directeur des programmes de BBC Alba, à propos de S4C, le budget d'une chaîne est en rapport avec la démographie linguistique: "Historically that was the reason they said, that for every Gaelic speaker there are 10 Welsh speakers so it's fearsome» ${ }^{48}$. Devant le peu d'évolution de leurs budgets et de leur audimat dans leurs espaces des lieux respectifs, la plupart des chaînes ont de plus en plus recours à la convergence, dont la définition est donnée par la Commission européenne:

La convergence peut se définir comme la fusion progressive des services de radiodiffusion traditionnels et d'Internet. Il en résulte de multiples possibilités de visionnage qui vont des téléviseurs à connectivité Internet ajoutée aux services audiovisuels fournis sur ordinateur de bureau ou portable, tablette ou tout autre terminal mobile, en passant par les décodeurs diffusant du contenu vidéo en mode OTT (Over The Top). Les consommateurs utilisent aussi leur tablette ou leur téléphone intelligent tout en regardant la télévision, par exemple pour en savoir plus sur ce qu'ils regardent ou pour interagir avec leurs amis ou avec le programme télévisé ${ }^{49}$.

L'ensemble des chaînes depuis trois ans développent donc progressivement l'offre de contenus sur d'autres supports numériques. Elles sont conscientes des possibilités qu'offrent la convergence et la télévision connectée pour les langues celtiques. Toutes, sauf BBC Alba, peuvent diffuser les émissions en langue celtique sur Internet dans le monde entier. La chaîne écossaise diffuse ses contenus sur Internet seulement au Royaume-Uni par suite du géo-blocage qui limite la diffusion des émissions à l'espace des lieux, c'est-à-dire à l'Écosse et à l'ensemble du Royaume-Uni. En effet, pour pouvoir offrir un contenu dans toute l'Union européenne, des fournisseurs de contenus comme les chaînes de télévision doivent obtenir l'autorisation des titulaires de droits (artistes, interprètes, producteurs, auteurs, etc..) et plus encore respecter la législation applicable dans l'ensemble des États membres.

Quelques données extraites des derniers rapports sont révélatrices. En 2016, le nombre d'accès à des émissions de TG4 sur plusieurs supports numériques s'élevait à 2080000 soit $37 \%$ de plus qu'en 2015. En 2017, à cause d'une forte concurrence, il était à 2200000 . Parallèlement, les réseaux sociaux en irlandais de la chaîne augmentaient de 606 \% pour atteindre $10200000^{50}$. Au Pays de Galles, en 2016/2017, le visionnement des émissions sur la télévision connectée ou les différents supports numériques est passé de 7644044 à 8197041 en 2017/2018. Les plateformes numériques de réseaux sociaux (Twitter, Facebook, YouTube) sont passées de 18000000 en 2016/2017 à 37000000 en 2017/2018 soit $91 \%$ d'augmentation ${ }^{51}$. BBC Alba présente une situation particulière en raison d'une

48. Entretien avec Alan Esslemont, Celtic Media Festival, St. Ives, 2 avril 2014.

49. Commission européenne, Livre vert. Se préparer à un monde audiovisuel totalement convergent: croissance, création et valeurs, Bruxelles, 24 avril 2013, COM(2013)231 final, p. 3, en ligne: http:// eur-lex.europa.eu/LexUriServ/LexUriServ.do?uri=COM:2013:0231:FIN:FR:PDF.

50. TG4, Annual Report 2016, p. 10; et TG4, Annual Report 2017, p. 11.

51. S4C, Annual Report and Statement of Accounts for the 12 Month Period to 31 March 2017, p. 22; et S4C, Annual Report and Statement of Accounts for the 12 Month Period to 31 March 2018, p. 48. 
diffusion limitée. Le visionnement des émissions sur le site de la chaîne s'élevait à 4100000 en 2017/2018 et à 4700000 en 2016/201752. Entre 2017 et 2018, seules les sessions sur Twitter et Facebook ont augmenté de $9 \%$ pour Twitter et de $15 \%$ pour Facebook ${ }^{53}$. En 2017/2018, Twitter et Facebook totalisent 1024000 sessions. La pénétration hebdomadaire de BBC Alba sur Facebook atteint 35000 sessions $^{54}$. L'accès limité à Internet par le haut débit, en particulier dans les Highlands, explique en partie ce déficit. Selon Maggie Cunningham, la directrice générale: «[...] there are still major deficits in provision. It is too much of a challenge for many of our core audience to access our content online - a situation which is unacceptable and must be rectified ${ }^{55}$. Au total les chaînes celtiques en Irlande et au Royaume-Uni, sauf BBC Alba, ont compris l'intérêt qu'elles avaient à diffuser leurs flux hors de leur territoire, dans un espace des flux de plus en plus concurrentiel.

France 3 Bretagne développe aussi l'offre numérique comme deux rapports récents l'ont conseillé: le rapport d'Anne Brucy ${ }^{56}$ et celui de Marc Schwartz ${ }^{57}$. En mai 2015, France 3 Bretagne a communiqué l'audimat pour les années 2014 et 2015 des émissions en langue bretonne, soit pour les premiers mois de l'année 2015: 12218 visites des pages d'émission (35225 en 2014) et 26476 pages d'émission vues $(82213 \text { en 2014 })^{58}$. Le conseil régional de Bretagne a fourni les trois derniers audimats de Brezhoweb pour les années 2014 à 2017. On remarque l'instabilité du nombre d'utilisateurs de la chaîne sur Internet, passés de 26431 en 2014/2015 à 16527 en 2016/2017. Le nombre de vues est passé de 980592 à 1367621 en 2016/2017. La diminution du nombre de sessions : de 51363 à 33972 en 2016/2017 s'explique par la diffusion d'un documentaire exceptionnel en 2014/2015 qui a déclenché énormément de connexions. Les émissions de Brezhoweb sont en breton et sont rarement soustitrées. Certaines émissions le sont en replay. Il reste que l'augmentation de l'audimat des télévisions et émissions en langue celtique va dépendre de deux paramètres et en premier lieu de l'attitude des utilisateurs des différents supports numériques.

Manuel Castells note «l'énorme disparité entre le taux d'utilisation d'Internet par les personnes de plus de 60 ans et par les personnes de moins de 30 ans ${ }^{59}$. Plus loin, il remarque à propos des adolescents: «Ils regardent des émissions entières sur

52. MG Alba, Annual Report and Statement of Accounts 2016/2017, p. 9; et MG Alba, Annual Report and Statement of Accounts 2017/2018, p. 42.

53. MG Alba, Annual Report and Statement of Accounts 2017/2018, p. 38.

54. Ibid., p. 40.

55. Maggie Cunningham, "Chairperson's Message», in MG Alba, Annual Report and Statement of Accounts 2016/2017, p. 10.

56. Anne Brucy, France 3, un avenir régional, $1^{\mathrm{er}}$ juillet 2014, p. 47, en ligne: https://www.vie-publique. $\mathrm{fr} /$ sites/default/files/rapport/pdf/144000377.pdf.

57. Marc Schwartz et al., France Télévisions 2020: le chemin de l'ambition, rapport du groupe de travail sur l'avenir de France Télévisions, février 2015, p. 88, en ligne: http://www.culturecommunication. gouv.fr/Ressources/Rapports/Rapport-sur-l-avenir-de-France-Televisions.

58. Entretien avec Maël Le Guennec, France 3 Bretagne, 16 mai 2015.

59. Manuel Castells, Communication et pouvoir, Paris, Éditions de la Maison des sciences de l'homme, 2013, p. 101-102. 
l'écran de leurs ordinateurs, mais aussi, de plus en plus, sur des appareils portables ${ }^{60}$. Le phénomène semble être le même partout. Daniel Cunliffe et Rhodri ap Dyfrig soulignent aussi, pour le gallois, que "young adults are moving their viewing habits to online ${ }^{61}$. Les données de différents pays confirment cette tendance. Au RoyaumeUni, selon Ofcom (Office of Communications), $30 \%$ des 16/24 ans utilisent une tablette ou un smartphone pour regarder des émissions ou des films ${ }^{62}$. En Irlande, c'est $15 \%$ de la population de plus de 16 ans qui regardent la télévision sur une plateforme audiovisuelle autre que le téléviseur ${ }^{63}$. Il faut souligner la désaffection des jeunes pour la télévision traditionnelle et un visionnage de plus en plus important de cette dernière sur plusieurs plateformes. En effet, ce sont les jeunes générations éduquées dans une culture numérique qui sont le plus susceptibles d'étendre le taux d'audience et les parts de marché des chaînes de télévision celtiques. Mike Cormack souligne l'importance de la jeune génération dans la situation critique où se trouvent les langues celtiques:

All of this emphasises the importance of the younger generation. In an endangered language situation, it is, of course, the upcoming generation which is the important one. But the way in which current developments are happening in digital media puts even more emphasis on this, it is the generation which is already growing with Facebook, YouTube and Wikipedia which will determine how Gaelic will survive in the new digital landscape $e^{64}$.

Ce constat met bien en lumière le lien entre les jeunes et les différentes plateformes numériques. Grâce à celles-ci, les chaînes de télévision vont être déterritorialisées. Manuel Castells a commenté ainsi la transformation de la télévision: "Television needed the computer to be free from the screen ${ }^{65}$. Pour étendre leur audimat dans l'espace des flux, au-delà de leur territoire, les chaînes en langue celtique ont de plus en plus recours au sous-titrage optionnel en anglais ou en français. En Irlande, le BAI a fixé les objectifs du sous-titrage en 2018 entre 57 et $63 \%$ des émissions diffusées ${ }^{66}$. Cependant, selon Pádhraic Ó Ciardha et mes propres mesures, $85 \%$ des émissions sont sous-titrées en anglais et $13 \%$ en irlandais ${ }^{67}$. D’après Ofcom, le pourcentage de sous-titrage en anglais atteint par S4C en 2016

60. Manuel Castells, Communication et pouvoir, p. 103.

61. Daniel Cunliffe, Rhodri ap Dyfrig, «The Welsh Language on YouTube: Initial Observations », in Social Media and Minority Languages..., p. 143.

62. Ofcom, Adults' Media Use and Attitudes. Report 2016, avril 2016, p. 61, en ligne: https://www. ofcom.org.uk/research-and-data/media-literacy-research/adults/adults-media-use-and-attitudes.

63. B\&A, TV Trends. A View from the Establishment Survey, 2017, p. 9, document consulté en ligne le 4 janvier 2017, désormais inaccessible.

64. Mike Cormack, "Gaelic in the New Digital Landscape», in Gaelic Communities Today, Gillian Munro, Iain Mac an Tàilleir (dir.), Edimbourgh, Dunedin Academic Press, 2010, p. 137.

65. Manuel Castells, The Information Age: Economy, Society, and Culture, vol. I, The Rise of the Network Society, $2^{\mathrm{e}}$ éd., Chichester, Wiley-Blackwell, 2010, p. 371.

66. Broadcasting Authority of Ireland, BAI Updates the Access Rules. Broadcasting Codes and Policy, Appendix 1, «Access Targets and Timeframes 2014-2018», p. 10, en ligne: http://www.bai.ie/en/ media/sites/2/2016/08/20160106_BAI_AccessRules2016_vFinal.pdf.

67. Entretien avec Pádhraic Ó Ciardha, directeur adjoint de TG4, 23 avril 2015. 
a été de $77,79 \%$ et de $66 \%$ pour BBC Alba ${ }^{68}$. Toutes les émissions en breton de France 3 Bretagne sont sous-titrées. Le Conseil supérieur de l'audiovisuel (CSA) a fixé pour Brezhoweb un volume d'heures sous-titrées à 80 heures soit $5 \%$ du volume total $^{69}$. Le sous-titrage en anglais ou en français augmente l'audimat d'une chaîne.

En effet, non seulement, les locuteurs sont concernés par le sous-titrage mais aussi les non-locuteurs. Selon Eithne O'Connell, les sous-titres en anglais ont leur place. Elle admet que leur utilisation peut se justifier: «English subtitles can make programmes in Irish accessible to the large sector of the Irish population, which would otherwise be excluded. On that basis, their use is justifiable ${ }^{70}$. Les chaînes doivent absolument justifier d'un bon indice d'écoute sur l'ensemble de la population si elles veulent continuer d'avoir son soutien et bénéficier d'un budget qui leur permette d'émettre $^{71}$. Cependant, ajoute-t-elle, les deux types de sous-titrages en langue minoritaire et en langue majoritaire ont des avantages et des inconvénients. Les sous-titres en langue minoritaire augmentent la visibilité de cette langue ainsi que son statut et son prestige. Ils peuvent ainsi participer à l'entretien et au développement de la langue minoritaire. D'autre part, il est exact que des sous-titres en anglais attirent des audiences plus grandes, stimulent l'intérêt, augmentent l'indice d'écoute et le soutien de la population à l'extérieur de la communauté linguistique. Un meilleur compromis est le sous-titrage en option, bien que ce dernier puisse être encore problématique pour certaines chaînes ${ }^{72}$. Il faut remarquer que la plupart des télévisions en langue celtique ont adopté le sous-titrage optionnel. Eithne O’Connell concède toutefois que, dans l'ensemble, les téléspectateurs irlandophones, gaélophones, ou gallophones, ne se sont pas beaucoup montrés hostiles à l'utilisation permanente des sous-titres en anglais. Ils ont préféré " a live and let live attitude», les émissions en langue minoritaire représentant un "fair compromise between the needs of competing linguistic groups ${ }^{73}$. La République d'Irlande, dans une moindre mesure l'Irlande du Nord, le Pays de Galles, l'Écosse et France 3 Bretagne ont tous opté pour le bilinguisme. Celui-ci est adopté par la plupart des chaînes de télévision celtiques. Dans la mesure où ces dernières sont, en partie, des entreprises commerciales, étendre l'audience au-delà de l'espace des lieux est devenu une nécessité. Il faut dire que la concurrence entre toutes les chaînes est rude dans le mediascape. Eithne O'Connell reconnaît que:

68. Ofcom, Television Channels Required to Provide Television Access Services in 2016, 22 juin 2015, document consulté en ligne en janvier 2015, désormais inaccessible.

69. Conseil supérieur de l'audiovisuel, Rapport relatif à l'accessibilité des programmes de télévision aux personnes handicapées et à la représentation du handicap, année 2013, avril 2014, p. 26, en ligne: https://www.csa.fr/Informer/Collections-du-CSA/Travaux-Autres-publications/Rapports-augouvernement.-parlement.-etc/Rapport-relatif-a-l-accessibilite-des-programmes-de-televisionaux-personnes-handicapees-et-a-la-representation-du-handicap-Annee-2013.

70. Eithne O'Connell, «Subtitles and Minority Language Broadcasting», 13 décembre 2014, en ligne: https://gaidhlig-tv.tumblr.com/post/104092170492/subtitles-and-minority-language-broadcasting-dr.

71. Ibid.

72. Eithne O’Connell, communication au colloque «Gaelic Broadcasting: Issues, Challenges and the Way Forward», 15 mars 2016.

73. Eithne O’Connell, «Subtitles and Minority Language Broadcasting», p. 3. 
The proliferation of digital channels and the likely attendant loss of content quality due to cost cutting, while presenting even more competition for cash-strapped minority language programming, may offer new possibilities for attracting larger transnational audiences to the specificity of much minority language output ${ }^{74}$.

La déterritorialisation des émissions en langue celtique n'est pas immédiatement évidente. Deux millions de membres de la diaspora en langue galicienne en Amérique du Sud peuvent regarder la Televisión de Galicia grâce au satellite, gonflant ainsi les chiffres de l'audience considérablement ${ }^{75}$. La diaspora irlandaise disséminée à travers le globe peut capter TG4 grâce à Internet. Eithne O'Connell avoue que "While Irish, as a much smaller minority language, does not have the same potential transnational audience ${ }^{76}$, la présence sur Internet de TG4, par exemple, lui confère une audience globale au-delà de la communauté linguistique virtuelle qu'elle a forgée dans les années récentes sur l'île d'Irlande ${ }^{77}$. Une chaîne diffusée sur le Web va, par ailleurs, acquérir une valeur symbolique de prestige et de modernité comme le souligne Pádhraic Ó Ciardha: «I think the language needed it to be associated with youth and cool and all those dreadful things ${ }^{78}$. Néanmoins, il reste difficile de prédire quelle sera la conséquence de la convergence des différentes plateformes sur les langues minoritaires ${ }^{79}$. La déterritorialisation des chaînes dans l'espace des flux est cependant engagée. TG4, S4C et Brezhoweb ouvrent la voie. Ofcom dans son rapport de 2015 écrit qu'il existe une tendance à regarder plus d'émissions sur les écrans autres que ceux de la télévision traditionnelle:

A shift towards non-linear: younger audiences consume less of their audio-visual content through linear methods than do other age groups. Our Digital Day research showed that only $50 \%$ of 16-24s' total audio-visual consumption (including DVDs, short-form, recorded $T V$, catch-up and other on-demand services, and traditional linear TV) and $61 \%$ of 25-34s' total consumption, is through linear television, compared to $69 \%$ for all adults ${ }^{80}$.

Le deuxième paramètre à prendre en compte pour augmenter l'audimat des chaînes celtiques est la diffusion sur le Web, dans l'espace des flux, d'œuvres non soumises au géo-blocage pouvant être vues et adaptées par d'autres pays. Les soap operas et les sitcoms sont de bons exemples car ils obéissent tous à des codes transnationaux comme l'a démontré Carmen Compte. Elle distingue un certain nombre d'invariants dans les soap operas et les sitcoms, où «les thèmes sont variés et évoluent avec la société comme par effet de miroir». Ces invariants,

74. Eithne O’Connell, «Towards a Template for a Linguistic Policy...», p. 188.

75. Ibid.

76. Ibid.

77. Ibid.

78. Entretien avec Pádhraic Ó Ciardha, directeur adjoint de TG4, 5 mai 2011.

79. Eithne O'Connell, «Towards a Template for a Linguistic Policy... », p. 189.

80. Ofcom, Public Service Broadcasting in the Internet Age, Ofcom's Third Review of Public Service Broadcasting, 2 juillet 2015, p. 19, en ligne: https://www.ofcom.org.uk/_data/assets/pdf_file/0025/63475/ PSB-statement.pdf. 
écrit-elle encore, "permettent d'évoquer une structure générale et les caractéristiques propres au genre ${ }^{81}$. C'est ainsi que l'on peut visionner en direct ou en replay sur tous les supports numériques la plupart des soap operas: Ros na Rún (TG4), Pobol y Cwm (S4C), Breizh Kiss (France 3 Bretagne) et Ken Tuch' (Brezhoweb). Si certains de ces soap operas et sitcoms intègrent une assez forte identité culturelle, ils ne suivent pas moins les conventions du genre. Un progrès a été accompli avec leur diffusion sur le Web dans l'espace des lieux et dans l'espace des flux ${ }^{82}$. Si BBC Alba ne diffuse toujours pas ses émissions hors des frontières du Royaume-Uni, il reste que son soap opera Bannan diffusé depuis 2015 « is also now poised for broadcast internationally, having been signed up by DRG, one of the leading independent distributors of programmes in the world» car, selon Chris Young, son producteur:

When we first embarked on producing Bannan, we were clear that the drama should be appealing not only to a Gaelic and Scottish audience but to have strong storylines that would resonate across borders ${ }^{83}$.

Il nous faut conclure que le passage de la télévision traditionnelle, territorialisée dans l'espace des lieux, à une télévision sur Internet, déterritorialisée dans l'espace des flux et sur plusieurs supports n'en est qu'à ses débuts. Déjà, certaines émissions en langue minoritaire peuvent être regardées sur Internet avec un soustitrage optionnel. Le sous-titrage en anglais optionnel est d'ailleurs la solution préférée par les chaînes pour développer leur part de marché et leur pénétration. George Jones l'a bien souligné: "Increasingly, as media develop their global reach by means of such devices as television on the internet, this applies also to speakers who live outside the country altogether ${ }^{84}$. Finalement, le passage des chaînes en langue celtique de la télévision linéaire à la télévision non linéaire va dépendre de l'évolution des langues qu'elles soutiennent, et d'éléments incontournables et parfois très fragiles. Parmi ceux-ci, on trouve ceux qui appartiennent à l'espace des lieux: le nombre de locuteurs, l'enseignement des langues minoritaires, l'attitude des gouvernements et des citoyens à l'égard des langues, le financement des chaînes et l'attitude des jeunes générations. Plus récemment se sont ajoutés des développements propres à l'espace des flux et au financescape, avec la mise en place du grand marché numérique unique préparé par la Commission européenne

81. Carmen Compte, "L'influence des soap opera sur les stratégies narratives des séries télévisées", Revue des interactions humaines médiatisées, vol. 9, n² 2, 2008, p. 12, en ligne: http://europia.org/ RIHM/V9N2/RIHM9(2)-2Compte.pdf.

82. Pierre Costecalde, Les télévisions celtiques (TG4, S4C, BBC Alba, France 3 Bretagne, Brezhoweb). De l'espace des lieux à l'espace des flux: territorialisation et déterritorialisation, thèse de doctorat, université Rennes 2, 2018.

83. «"Bannan” Goes Global», West Highland Free Press, 22 septembre 2016, en ligne: https://www. whfp.com/2016/09/22/new-series-of-bannan-this-week/.

84. George Jones, «Minority-Language Audiovisual Media in Europe», communication au $1^{\text {er }}$ congrès sur le journalisme basque, Bilbao, 9-12 novembre 2004, p. 10, en ligne: http://citeseerx.ist.psu. edu/viewdoc/download?doi=10.1.1.502.9085\&rep=rep1\&type=pdf. 
ainsi que les discussions préalables au Brexit. Divina Frau-Meigs attire justement l'attention sur certaines valeurs que véhiculent les flux financiers du financescape et qui dominent toutes les autres: "Que la stratégie soit nationale ou globale, ce sont les valeurs commerciales aux pressions privatisantes qui tendent à l'emporter, avec peu de considération pour l'intérêt public » ${ }^{85}$ et, pouvons-nous ajouter, pour les valeurs culturelles. On retrouve ici la domination du financescape sur tous les autres paysages.

Il demeure que l'avenir des chaînes de télévision en langue minoritaire dépend surtout de l'utilisation que vont en faire les nouvelles générations. Manuel Castells exprime ainsi sa confiance en celles-ci:

La Toile a déjà transformé la télévision. Les adolescents interviewés par des chercheurs du Centre de l'avenir numérique (Center for the Digital Future) de l'université de la Californie du Sud ne comprennent même pas pourquoi il faudrait s'adapter à des horaires imposés pour pouvoir regarder la télévision. Ils regardent des émissions entières sur l'écran de leurs ordinateurs, mais aussi, de plus en plus, sur des appareils portables. Ainsi, même si la télévision continue d'être un média de masse très important, sa diffusion et son format sont en train d'être transformés, par la personnalisation de sa réception (Center for the Digital Future, «World internet Survey», diverses années; Cardoso, 2006 $\left.{ }^{86}\right)^{87}$.

Les chaînes de télévision celtiques sur Internet et les réseaux sociaux sont une chance pour les langues minoritaires qui passent ainsi de l'espace des lieux à l'espace des flux, de la territorialisation à la déterritorialisation. Le numérique élargit les potentialités des langues celtiques. En effet, faire de la télévision est d'abord une question financière, et Maël Le Guennec cite l'exemple de longs-métrages tournés par France 3 qui coûtent 10000 euros la minute ${ }^{88}$. Pour survivre, une chaîne en langue minoritaire doit obligatoirement étendre son audience et ainsi accroître son budget et sa crédibilité. Un système optionnel de sous-titrage en anglais ou en français ou dans la langue celtique de son choix semble être un des paramètres à considérer, tout comme le sont les futurs accords sur le Brexit et le développement du grand marché numérique. Le Livre vert publié en 2013 par la Commission européenne sur la convergence des moyens audiovisuels résumait la situation ainsi :

En Europe, l'expérience des consommateurs en matière de services audiovisuels fournis en ligne se résume encore, la plupart du temps, à un choix limité de programmes et à un accès refusé pour des raisons de limites géographiques. Les applications sur téléviseur intelligent sont souvent bridées par des paramètres nationaux et la présélection des fabricants, et l'accès au contenu d'autres pays de l'UE est généralement bloqué.

85. Divina Frau-Meigs, "Médias", in Dictionnaire critique de la mondialisation, Cynthia GhorraGobin (dir.), Paris, A. Colin, 2012, p. 401.

86. Gustavo Cardoso, The Media in the Network Society: Browsing, News, Filters and Citizenship, Lisbonne, Center for Research and Studies in Sociology, 2006.

87. Manuel Castells, Communication et pouvoir, p. 103.

88. Entretien avec Maël Le Guennec, France 3 Bretagne, 4 octobre 2014. 
La technologie permettra de surmonter ces obstacles. Les producteurs de contenu, les sous-titreurs et les chercheurs ont commencé à nouer des alliances pour partager les ressources et outils linguistiques disponibles $[\ldots]^{89}$.

Dès la fin des années 1960, Michel Foucault (cité par Sari Pietikäinen et Helen Kelly-Holmes ${ }^{90}$ ) dressait une description de l'espace à l'époque présente qui anticipait les conditions de la globalisation et la déterritorialisation des flux et des réseaux:

Nous sommes à l'époque du simultané, nous sommes à l'époque de la juxtaposition, à l'époque du proche et du lointain, du côte-à-côte, du dispersé. Nous sommes à un moment où le monde s'éprouve, je crois, moins comme une grande vie qui se développerait à travers le temps que comme un réseau qui relie des points et qui entrecroise son écheveau ${ }^{91}$.

C’est donc bien ce que signifie Súil Eile (un autre regard), le logo de TG4 créé par Pádhraic Ó Ciardha, qui est une autre façon d'exprimer la déterritorialisation et l'ouverture, objectifs que toutes les chaînes en langue celtique s'efforcent de réaliser.

Pierre CostecAlde

Lycée Jeanne d'Arc, Millau

89. Commission européenne, Livre vert. Se préparer à un monde audiovisuel totalement convergent..., p. 6 .

90. Helen Kelly-Holmes, Sari Pietikäinen, «The Peripheral Multilingualism Lens. A Fruitful and Challenging Way Forward?», in Multilingualism and the Periphery, Sari Pietikäinen, Helen Kelly-Holmes (dir.), Oxford, Oxford University Press, 2013, p. 217.

91. Michel Foucault, "Des espaces autres» (conférence au Cercle d'études architecturales, 14 mars 1967), Architecture, mouvement, continuité, no 5, octobre 1984, p. 46-49, en ligne: http://1libertaire. free.fr/Foucault12.html. 